\title{
Características del Self en Pacientes con Trastorno Obsesivo Compulsivo: una aproximación desde el Enfoque Integrativo Supraparadigmatico
}

\section{Self Features in Patients with Obsessive Compulsive Disorder: an approach from Supraparadigmatic Integrative Model}

\author{
Texia Bejer Tajmuch ${ }^{1}$ \\ Universidad Santo Tomás, Santiago, Chile \\ (Rec: febrero 2015 - Acept: junio 2015)
}

\begin{abstract}
Resumen
En la presente investigación, se intenta comprender el trastorno Obsesivo Compulsivo (TOC) más allá de los síntomas, como una forma de planear estrategias de intervención diferentes y que ayuden a aliviar el sufrimiento de los pacientes. Este trastorno presentaría una prevalencia aproximada entre un $1.5 \%$ y un $3 \%$ con variaciones en diferentes ciudades, por lo que es alta la presencia de él, sin embargo, la consulta por éste es baja, ya que el trastorno en sí conlleva vergüenza y miedo, afectando así a la persona en su identidad, en como el sujeto se aproxima al mundo, como desea que la vida sea para él. Se toma el marco referencial del Enfoque Integrativo Suprapradigmático, especialmente las funciones del Self propuestas por éste enfoque. La investigación es descriptiva y pretende caracterizar las funciones del Self de pacientes con TOC, la muestra es de 18 pacientes de un centro de atención psicológica de la ciudad de Santiago de Chile y que responden la Ficha de Evaluación Clínica Integral (FECI).
\end{abstract}

Palabras clave: TOC, EIS, funciones del self.

\begin{abstract}
In this research, we try to understand Obsessive Compulsive Disorder (OCD) beyond its symptoms, as a way of planning different intervention strategies to help alleviate the suffering of patients. This disorder has a prevalence between $1.5 \%$ and $3 \%$ with variations in different cities. Despite its high prevalence, consultation levels are low since the disorder itself is associated with shame and fear, affecting a person's identity, the way the subject approaches the world and the life wished for. The reference frame adopted is the Suprapradigmatic Integrative Model, especially the functions of the Self proposed by this approach. The research is descriptive and aims to characterize the functions of the Self of patients with OCD. The sample is of 18 patients attended at a Psychological Services Center in Santiago, Chile, evaluated with the Integral Clinical Evaluation (FECI).

Keywords: OCD, EIS, Functions of Self.
\end{abstract}

\footnotetext{
1 Correspondencia a: Texia Bejer Tajmuch. Universidad Santo Tomás, Facultad de Ciencias Sociales, Ejército Libertador \#146, Región Metropolitana de Santiago, Chile. Tel.: (56-02) 2362 5000. E-mail: texiabejer@santotomas.cl.
} 


\section{Introducción}

Dentro de los trastornos de ansiedad existen cuadros clínicos que son más conocidos, como el trastorno de pánico, las fobias, la ansiedad social, etc.; sin embargo, el trastorno obsesivo compulsivo presenta poca mención tanto por los que sufren de él como por los que lo tratan, conjuntamente aparecen pocos desarrollos modernos en torno al tratamiento. Es como si la "vergüenza" que reportan los sujetos que padecen este trastorno, se extendiera de una manera extraña a quienes dan atención a dichos pacientes; al mismo tiempo, los tratamientos propuestos aparecen centrados solo en los síntomas, no logrando ver de manera íntegra al ser humano que lo sufre.

En la actualidad se habla del trastorno obsesivo compulsivo (TOC) y su espectro, haciendo referencia a diferentes cuadros asociados, que comparten bases biológicas y fenomenología similar (Cía, 2002) y cuya base, la ansiedad desadaptativa, quita libertad y capacidad de decisión a los seres humanos.

En materias de comprensión y tratamiento, se ha avanzado desde el siglo XVII, en donde se encuentran las primeras descripciones de síntomas TOC y los primeros tratamientos a través del exorcismo.

A comienzos del siglo XIX pasa desde esta mirada espiritual a una médica, con Esquirol considerado un pionero con la descripción de lo que él denominó monomanía, que ya incluía lo que actualmente se conoce por TOC. A mediados del siglo XIX aparecen las descripciones de Wesphal quien subrayó la presencia de ideas que irrumpen en la consciencia a pesar de los esfuerzos de la persona por resistirlas y además detectó la presencia de síntomas psicóticos en los casos de TOC más graves, ilustrando con esto una importante polémica relacionada con su diagnóstico diferencial, polémica que en alguna medida se mantiene hasta hoy. Finalmente fueron Janet en 1903 y Schneider en 1925 quienes lo ubicaron en el terreno de las neurosis principalmente por su carácter egodistónico, la lucha ansiosa del psiquismo y por la experiencia subjetiva de compulsión. (Downey, 2006, p. 6)

Desde el siglo XX, el cuadro es descrito en manuales diagnósticos de salud mental (DSM y CIE), y su tratamiento está centrado en el área médica (por medio de la farmacoterapia) y en algunas líneas iniciales en psicoterapia. Es por esto que se han desarrollado avances desde la concepción de "posesión demoniaca" a elementos biológico-psicológicos que se encontrarían en su base. Sin embargo, aún se pueden observar altos niveles de prejuicios frente a los pacientes que sufren esta enfermedad y su espectro. Todo ello acompañado de una baja tasa de consulta y una alta tasa de recaída.

En los últimos años, se ha ido desarrollando en la psicología chilena, un enfoque llamado Enfoque Integrativo Supraparadigmático (EIS, en adelante), propuesto por el Dr. Roberto Opazo y su equipo (Opazo, 2001). En él, se plantea que la comprensión de los fenómenos psicológicos y mentales, requiere de una amplia mirada al mundo psíquico del sujeto. De este modo, el ser humano es concebido como un sujeto que conoce un mundo objetivo desde una construcción personal y única, siendo capaz de psicofacturar los estímulos para adaptarse al entorno. Así pues, el EIS propone que en la comprensión de cada ser humano, habría que considerar los aspectos biológicos, afectivos, cognitivos e inconscientes, y a la vez considerarlo inserto y partícipe dentro de diversos ambientes (familia, grupos, sociedad, etc.). Es por esto que propone seis paradigmas (biológico, afectivo, cognitivo, inconsciente, ambientalconductual y sistémico) que son integrados en el self, el cual actúa como eje central, teniendo diferentes funciones. A saber: función de identidad, función de significación, función de auto-organización, función de búsqueda de sentido, función de conducción de vida y función de toma de conciencia que permitirían al ser humano interactuar consigo mismo y con el entorno (Fernández-Álvarez \& Opazo, 2004).

Dentro del EIS, el self se convertiría en el eje central que coordina y reúne todas aquellas características individuales, y que hacen único al ser humano. Así, las funciones del self regularían la forma en que el ser humano está en el mundo y se relaciona tanto consigo mismo como con el entorno. Desde esta perspectiva "integrativa" del EIS, se analizarán las diferentes funciones del self en pacientes con TOC.

\section{Antecedentes}

\section{Trastorno Obsesivo Compulsivo}

Según la definición del DSM-IV, este trastorno se ubicaría dentro de los trastornos de ansiedad. Sin embargo, en el DSM-V, el TOC se encuentra en un apartado especial: "Trastorno obsesivo compulsivo y desórdenes relacionados". Esto da cuenta de que 
este tipo de trastorno cobra importancia al momento de diagnosticarlo y tratarlo (American Psychological Association [APA], 2013). El TOC se caracterizaría por la presencia de obsesiones, compulsiones o ambas; las cuales constarían de pensamientos, impulsos, imágenes intrusivas y comportamientos o actos mentales cuyo objetivo es reducir la ansiedad causada por esos pensamientos. Este trastorno es lo suficientemente grave como para provocar una marcada perturbación, pérdidas de tiempos significativos, acusado deterioro de la actividad general o malestar clínicamente significativo; no debiéndose a los efectos fisiológicos directos de una medicación, sustancia o condición médica general. Además, en el DSM-V se hace distinción según el sujeto presente "bueno, regular, pobre o ningún insight sobre sus pensamientos intrusivos" (APA, 2013, p. 237).

Por otro lado, este trastorno presentaría una prevalencia a nivel mundial aproximadamente de $2.5 \%$ (Antony, 1998) aunque los últimos cálculos indican que se aproximaría al 3\% en población en general (Asociación del trastorno obsesivo compulsivo, 2015). En Chile, tan solo hay una investigación que hace mención a la prevalencia del TOC, pero restringida solo al área del Gran Santiago. En dicha investigación, aparecería una prevalencia del 1.3\% (Araya, Rojas, Fritsch, Acuña \& Lewis, 2001; Cía, 2006).

Por otro lado, es común que este tipo de pacientes presente co-morbilidad con otros trastornos psiquiátricos. Aunque el trastorno depresivo es el más frecuente, también aparecen otros trastornos de ansiedad asociados a este mal (Torres et al., 2014).

Por otro lado, aunque habría algunas teorías psicológicas sobre el origen del TOC, se pueden encontrar pocos tratamientos efectivos para dicho trastorno.

Aunque aparece cada vez con más frecuencia, es posible observar avances en el ámbito de la farmacoterapia para el tratamiento del TOC. Siendo los tratamientos conductuales y cognitivo conductuales, que surgen entre los años sesenta y setenta, los que presentan estrategias avaladas a través de investigaciones, con cierta efectividad en la disminución de los síntomas y en la consecución de alivio para los pacientes que presentan TOC clásico, es decir, obsesiones y compulsiones. Sin embargo, aparecen otros cuadros del espectro de TOC, por ejemplo, solo obsesiones o compulsiones mentales, en los que las técnicas mencionadas, son poco o nada efectivas. En dichos casos la neurobiología toma el control y aparece la neurocirugía como una opción (Downey, 2006).
Sin embargo, se pueden observar estrategias de tratamiento desde otras líneas de la psicología, como apoyo al tratamiento tradicional. En este sentido, desde la línea sistémica-familiar, muchos autores resaltan las ventajas de realizar intervenciones en que se incluyan a los otros significativos en el tratamiento, además de la incorporación de grupos de apoyo tanto para pacientes como para familiares, en donde se les enseñan técnicas de enfrentamiento basadas en la psicoeducación del diagnóstico, tratamiento, medicación, etc. Esto centrado en ubicar al familiar como un coterapeuta activo en el tratamiento y también, en la prevención de recaídas (Marks, Hodgson \& Rachman, 1975).

Desde el EIS, se han analizado las diferentes influencias etiológicas al momento del inicio y mantención del cuadro clínico TOC. Así, se postulan, desde los diferentes paradigmas, fuerzas etiológicas que se conjugan en cada persona para configurar el cuadro de manera única. Y de la misma manera afectarían el self de la persona que sufre este trastorno, viéndose alteradas todas sus funciones, pero en "ponderaciones" diferentes, apareciendo más alteradas las funciones de significación, conducción existencial y autorganización (Downey, 2006).

\section{Metodología}

La investigación se realizará con metodología mixta, en un diseño no experimental, descriptivo y transversal. La muestra es no probabilística, realizándose una selección intencionada de sujetos tipo que queda conformada por 18 pacientes que son atendidos entre los años $2000 \mathrm{y}$ $2007^{2}$, en un centro de atención psicológica de Santiago de Chile. ${ }^{3}$. La distribución es de $44.4 \%$ hombres y $55.6 \%$ mujeres, cuyas edades van entre 18 y 49 años. Todos ellos diagnosticados con TOC, según los criterios diagnósticos del DSM-IV, no presentando alteración del examen ni juicio de realidad (en el DSM-V con regular y buen insight). Adicionalmente, contestaron el cuestionario que se utiliza en el centro de atención psicológica de la ciudad de Santiago de Chile, la Ficha de Evaluación Clínica Integral (FECI). El FECI es un cuestionario de autoreporte que además contiene escalas clínicas, algunas validadas internacionalmente y otras validadas en el centro de atención psicológica de la ciudad de Santiago

\footnotetext{
2 Los sujetos de la muestra cumplen con los requisitos necesarios para poder analizar lo que se desea. Habiendo firmado consentimiento informado para participar en investigaciones en ICPSI.

3 En el análisis se utilizaron el Chi cuadrado con un nivel de confianza de $95 \%$.
} 
de Chile, además contiene ítems y escalas Likerts que dan cuenta de diferentes aéreas del sujeto (Bejer, 2010).

Se analizaron 84 variables, dentro de las cuales se encuentran las escalas del $\mathrm{EPQ}^{4}$, autoimagen, autoeficacia, autoestima, satisfacción de necesidades, satisfacción matrimonial, asertividad, alexitimia, repertorio conductual, perfeccionismo, ansiedad, depresión, tolerancia a la frustración y de vulnerabilidad biológica (Bejer, 2010). Adicionalmente, se analizaron otras variables tales como edad, descripción de sí mismo, escala de rasgos de personalidad, capacidad de empatía, capacidad de disfrutar, escala de dificultades en la toma de decisiones, expectativas de cambio, propensión emocional, etc. La idea era cubrir las diferentes áreas de funcionamiento del sujeto.

Además, en el FECI aparece un ítem en donde se le solicita al sujeto describirse con palabras. Este ítem, se codificó y se categorizó, obteniendo así adjetivos que daban cuenta de la descripción de sí mismo en cada sujeto de la muestra.

El análisis de los datos se realizó con el programa SPSS 17 (2008).

\section{Resultados}

Para efectos del análisis se agruparán los resultados en: self biológico (entendido como la predominancia de aspectos biológicos al activarse las diversas funciones del self), función de identidad, función de significación, función de autorganización y función de conducción de vida.

Tabla 1

Paradigma biológico

\begin{tabular}{lcc}
\hline \multicolumn{1}{c}{ Variable } & $\%$ & $\mathrm{p}$ \\
\hline Ant. Psiquiátricos familiares & $44 \%$ & .005 \\
Neuroticismo alto & $77.8 \%$ & .018 \\
Vulnerabilidad biológica alta & $61.1 \%$ & .045 \\
Sueño poco reparador & $88.9 \%$ & .002 \\
Problemas en el desarrollo & $38.9 \%$ & .001 \\
Comorb. T. Pánico & $16.7 \%$ & .005 \\
Comorb. Abuso sustancias & $5.6 \%$ & .000 \\
\hline
\end{tabular}

$n=18$

\footnotetext{
4 Cuestionario de Eysenck
}

En la tabla $\mathrm{N}^{\circ} 1$ se observa que de los pacientes con TOC hay un $44 \%$ con familiares con algún trastorno psiquiátrico, lo que es concordante con lo que se observa en diferentes investigaciones de este trastorno. Aunque en la muestra seleccionada no aparecerían familiares que reportan TOC, en estudios realizados en Argentina, se menciona que "el 35\% de los familiares de primer grado de pacientes con TOC, de inicio en la infancia, resultan afectados del trastorno" (Cía, 2006, p. 115). Además, se puede observar en los estudios llevados a cabo por Nicolini, Weissbecker, Mejia \& de Carmona (1993) que el riesgo relativo de presentar TOC si existen familiares que lo presentaban, es de 4 a 5 veces mayor. De este modo, aparece la confirmación de que existiría un componente familiar, hereditario en la base del TOC (Nicolini et al., 1993).

Conjuntamente, se puede observar que el $77 \%$ de los paciente de la muestra presentan un valor alto en la escala de Neuroticismo de Eysenck, y el 61.1\% presentaría alta vulnerabilidad biológica. Estos datos pueden dar cuenta de que, en dichos pacientes, aparecería desde los aspectos biológicos, mayor predisposición a la inestabilidad emocional, así como también a un predominio de emociones displacenteras (Opazo, 2001).

Por otro lado, el $88.9 \%$ presenta sueño poco reparador, así como el $38.9 \%$ presentaría algún tipo de alteración durante el desarrollo. Conjuntamente, se puede observar que hay un $16.7 \%$ que presentaría co-morbilidad con trastorno de pánico y $5.6 \%$ con abuso de sustancias. No apareciendo una asociación significativa con trastorno depresivo. Esto último es algo contradictorio a estudios de co-morbilidad en pacientes con TOC, los que, aunque varían dependiendo de las muestras clínicas y de los criterios y métodos para medir el desorden depresivo, indican que al menos "un tercio de los individuos con TOC tiene historia significativa de depresión" (Cía, 2006, p. 100).

En la tabla $\mathrm{N}^{\circ} 2$ se puede observar que el $61.3 \%$ se define en su infancia como un niño regalón, y que los valores que les han sido transmitidos son en el $16.7 \%$ rígidos y en el $16.7 \%$ contradictorios. Como se menciona anteriormente, habría una tendencia a que los pacientes con TOC tengan familiares en primer grado (padre, madre o hermanos) con dicho trastorno. De esta manera, la transferencia de los valores podría estar dándose a través de la socialización y el modeling; bases para que se desarrolle el trastorno. Sería interesante ver si dentro de este $16.7 \%$ se encontrarían pacientes con inicio en la infancia o inicio precoz, generando así, base para estructuras subyacentes profundas que determinen la identidad 
con elementos en la línea de lo obsesivo. Dentro de esto, no es extraño escuchar a pacientes con TOC referirse a sí mismos como "siempre he sido maniaco" (hombre, 22 años) o "yo siempre he sido así, no me gustan las cosas chuecas o sin definir" (mujer, 27 años).

Tabla 2

Función de identidad

\begin{tabular}{lcc}
\hline \multicolumn{1}{c}{ Variable } & $\%$ & $\mathrm{p}$ \\
\hline Autoefic. Laboral alta & $83.3 \%$ & .000 \\
"Desearía sentir más aprecio por mí mismo" & $83.3 \%$ & .000 \\
Autoestima baja & $66.7 \%$ & .006 \\
Regalón & $61.3 \%$ & .016 \\
Autoefic. Sexual alta & $61.1 \%$ & .042 \\
Autoefic. Intelectual alta & $61.1 \%$ & .016 \\
"Puedo hacer las cosas tan bien como la & $61.1 \%$ & .011 \\
mayoría de la gente" & & \\
V.T. Rígido & $16.7 \%$ & .001 \\
V.T. Contradictorio & $16.7 \%$ & .001 \\
\hline$n=18$ & & \\
\hline
\end{tabular}

Por otro lado, la autoeficacia general no aparece con alguna asociación significativa. Sin embargo, la autoeficacia en áreas específicas logra aparecer con significación. Así, el $83.3 \%$ se siente muy capaz de desempeñarse en el área laboral, el $61.1 \%$ se siente muy capaz de desempeñarse en el área intelectual y el $61.1 \%$ se siente muy capaz de desempeñarse en el área sexual. Tendiendo a predominar en un $61.1 \%$ la afirmación "puedo hacer las cosas tan bien como la mayoría de la gente". Esto también puede tener que ver con la forma en que tienden a evaluarse frente a la percepción de amenaza. En este sentido, a pesar de que pueden sentir que "son capaces" de desenvolverse de manera adecuada en la vida, frente a la sensación de amenaza (idea obsesiva) aparece muy baja autoeficacia, necesitando generar un control sobre esto y surgiendo así, las compulsiones.

En cuanto a la escala de autoestima, esta se presenta baja en el $66.7 \%$, tendiendo a predominar la afirmación "desearía sentir más aprecio por mí mismo". Esto daría cuenta de que la mayoría de los sujetos con TOC sentirían bajo aprecio por sí mismo o al menos un menor aprecio del que les gustaría, lo cual puede ser un aspecto importante al momento de plantearse un plan terapéutico frente a pacientes con TOC.

Tabla 3

Rasgos de personalidad

\begin{tabular}{lcc}
\hline \multicolumn{1}{c}{ Dimensiones } & $\%$ & $\mathrm{p}$ \\
\hline Honesto - Deshonesto (honesto) & $88.9 \%$ & .001 \\
Responsable - Irresponsable (responsable) & $66.7 \%$ & .006 \\
Inteligente - Poco Inteligente (inteligente) & $66.7 \%$ & .006 \\
Trabajador - Flojo (trabajador) & $66.7 \%$ & .009 \\
Simpático - Antipático (simpático) & $61.1 \%$ & .030 \\
Inestable & $27.8 \%$ & .029 \\
Cariñoso & $22.2 \%$ & .018 \\
Inteligente & $22.2 \%$ & .018 \\
Irritable & $22.2 \%$ & .000 \\
Perseverante & $22.2 \%$ & .018 \\
Responsable & $22.2 \%$ & .018 \\
Tímido & $22.2 \%$ & .018 \\
Agresivo & $16.7 \%$ & .005 \\
Alegre & $16.7 \%$ & .005 \\
Perfeccionista & $16.7 \%$ & .002 \\
Simpático & $11.1 \%$ & .001 \\
\hline$n=18$ & &
\end{tabular}

En la tabla $\mathrm{N}^{\circ} 3$, los sujetos tienden a definirse, por un lado, como honestos, responsables, inteligentes, trabajadores y simpáticos, en la escala de rasgos dada por el cuestionario. Por otro lado, en el momento de definirse ellos mismos, aparecen otros adjetivos que se pueden categorizar en "positivos y negativos". Así, en los aspectos "positivos" aparecen: cariñoso, inteligente, perseverante, responsable, alegre, simpático. En los aspectos "negativos" se pueden encontrar: inestables, irritables, tímidos y agresivos. En cuanto al rasgo de perfeccionista, el $16.7 \%$ lo marca como significativo. Según los estudios habría un $25 \%$ de los sujetos con TOC que presentarían co-morbilidad con trastorno de personalidad obsesiva (Cía, 2006). Llama la atención que los sujetos, al responder en el cuestionario que es parte del FECI, entregan las características positivas, aunque también aparecen características "negativas" que no son significativas. Sin embargo, cuando deben definirse desde sus propias 
palabras, surgen mayores adjetivos "negativos" que "positivos". Así, se puede observar que una paciente de 27 años, al definirse refiere: "soy organizada, mañosa, responsable, mal genio". Sin embargo, al indicar rasgos del FECI, aparecen rasgos en la línea activa, pacifica, tolerante.

\section{Tabla 4}

Función de significación

\begin{tabular}{lcc}
\hline \multicolumn{1}{c}{ Variable } & $\%$ & $\mathrm{p}$ \\
\hline Frecuente pensamiento rumiativo & $94.4 \%$ & .000 \\
Locus de control interno & $88.9 \%$ & .001 \\
Magnificación de lo negativo & $83.3 \%$ & .005 \\
"Constantemente me exijo perfección en & $83.3 \%$ & .005 \\
las cosas que hago" & & \\
Alta autoexigencia & $77.8 \%$ & .000 \\
"Me siento demasiado mal cuando alguien & $77.8 \%$ & .000 \\
me rechaza" & & \\
Escala perfeccionismo alta & $66.7 \%$ & .006 \\
Escala irracionalidad cognitiva & $66.7 \%$ & .011 \\
Atención selectiva a lo negativo & $44.4 \%$ & .042 \\
Valores: rígidos & $16.7 \%$ & .001 \\
\hline
\end{tabular}

En la tabla $\mathrm{N}^{\circ} 4$ se puede observar que el $94.4 \%$ de la muestra frecuenta pensamiento rumiativo, unido con locus de control interno (88.9\%). Además, el $83.3 \%$ presenta la distorsión cognitiva de magnificación de lo negativo (Ruiz, 1994). De la misma manera, el $83.3 \%$ también se identifica con la afirmación "constantemente me exijo perfección en lo que hago", lo que es consistente con que el $94.4 \%$ de la muestra puntúe alto en la escala de perfeccionismo. Es decir, los pacientes con TOC tienden a establecer altos estándares de desempeño en combinación con una autoevaluación excesivamente crítica de los mismos y una creciente preocupación por cometer errores (Bejer, 2010). También se puede observar que el $66.7 \%$ presenta un tipo de procesamiento cognitivo sobre sí mismo y sobre los demás (irracionalidad cognitiva alta), que puede producir desajustes emocionales y/o conductuales que son frecuentemente desadaptativos. Sin embargo, solo el $16.7 \%$ presentaría valores rígidos.
Al mismo tiempo, al hacer el análisis de los ítems del cuestionario EPQ, se encontraron preguntas significativamente importantes para los sujetos. De este modo, el $77.8 \%$ se detiene a pensar las cosas antes de hacerlas, al $88.9 \%$ le preocupa cosas que debió haber dicho o hecho, el $88.9 \%$ está permanentemente preocupado por algo y al $83.3 \%$ le preocupa su salud y/o cosas terribles que puedan suceder.

Tabla 5

Función de autorganización

\begin{tabular}{lcc}
\hline \multicolumn{1}{c}{ Variable } & $\%$ & $\mathrm{p}$ \\
\hline Se define como muy emotivo & $94.4 \%$ & .000 \\
$\begin{array}{l}\text { Se preocupa mucho tiempo luego de tener } \\
\text { una experiencia desagradable }\end{array}$ & $88.9 \%$ & .001 \\
Baja tolerancia a la frustración & $77.8 \%$ & .018 \\
Alta propensión a emociones displacenteras & $77.8 \%$ & .018 \\
Presencia de eventos estresantes & $66.7 \%$ & .004 \\
Frente a frustración reacciona con rabia & $66.7 \%$ & .006 \\
Frente a frustración reacciona con tristeza & $66.7 \%$ & .009 \\
Se afecta mucho frente a las críticas & $66.7 \%$ & .006 \\
Presencia de agresión física a terceros & $27.8 \%$ & .005 \\
\hline
\end{tabular}
$n=18$

En la tabla $\mathrm{N}^{\circ} 5$ se observa que el $94.4 \%$ de los sujetos reconocen ser muy emotivos, tendiendo a predominar emociones displacenteras tales como pena $(77.8 \%)$, rabia $(55.6 \%)$, angustia $(77.8 \%)$ y vergüenza (66.7\%). Apareciendo solo la ternura (66.7\%) como emoción placentera. Además, el $88.9 \%$ de los sujetos reconoce permanecer afectado por mucho tiempo después de vivenciar una experiencia displacetera y el $77.8 \%$ indica presentar alta propensión a dichas emociones. Sumado a esto, aparece que el $77.8 \%$ presenta baja tolerancia a la frustración, tendiendo a manifestar como reacción a esto rabia $(66.7 \%$ ) y tristeza (66.7\%). Conjuntamente, el 66.7\% reconoce afectarse mucho emocionalmente frente a las críticas y el $27.8 \%$ indica haber presentado algún tipo de episodio en que aparece agresión física a terceros. 
Tabla 6

Función de conducción de vida

\begin{tabular}{lcc}
\hline \multicolumn{1}{c}{ Variable } & $\%$ & $\mathrm{p}$ \\
\hline $\begin{array}{l}\text { No soporto hacer algo en forma mediocre } \\
\text { (mucho) }\end{array}$ & $77.8 \%$ & .000 \\
$\begin{array}{l}\text { Alta satisfacción de necesidades básicas } \\
\text { En toma de decisiones, excesivamente }\end{array}$ & $77.8 \%$ & .000 \\
$\begin{array}{l}\text { perfeccionista } \\
\text { Alta necesidad de saber }\end{array}$ & $72.2 \%$ & .002 \\
$\begin{array}{l}\text { Poca actividad física } \\
\text { En toma de decisiones, no mide }\end{array}$ & $55.7 \%$ & .009 \\
consecuencias & $66.7 \%$ & .009 \\
Cdta. agresión terceros & .030 \\
Cdta. destrucción objetos & $27.8 \%$ & .005 \\
\hline$n=18$ & $11.1 \%$ & .001 \\
\hline
\end{tabular}

En la tabla $\mathrm{N}^{\circ} 6$ se observa que el $77.8 \%$ afirma estar de acuerdo en que "no soportan hacer algo de manera mediocre", esto también se puede observar en que al momento de tomar decisiones aparecen dificultades al ser excesivamente perfeccionista $(72.2 \%)$ y no medir las consecuencias de lo que deciden (5.6\%).

Además, aparece el $66.7 \%$ con poca actividad física, tendiendo a mostrar dificultad para activarse. Por otro lado, aunque el $77.8 \%$ indica tener satisfechas sus necesidades básicas, el $66.7 \%$ indica la presencia de la necesidad de saber. Asimismo aparece un porcentaje de sujetos $(27.8 \%)$ que manifiesta haber presentado algún tipo de conducta de agresión a otros y un $11.1 \%$ reconoce conductas de destrucción de objetos.

\section{Conclusiones}

Como los estudios y las investigaciones indican, se ha observado que en el TOC aparece un alto compromiso biológico. Así pues, los resultados obtenidos dan cuenta de elementos de base biológica que afectarían, al momento de activarse cada una de las funciones del self. De esta manera, los diferentes paradigmas (cognitivo, afectivo, incluso los elementos más inconscientes) se verían afectados por dichos elementos biológicos al momento de estar con el TOC. Esto mismo, llevaría a que el sujeto presentara tendencia a mirar al mundo y a sí mismo de una manera específica, el Dr. Soria, en 2006, plantea que:

Los pacientes con TOC, por su hiperreactividad cortico frontal sufrirían incrementos en su sentido del juicio (ideas de culpa), manifestarán respuestas impregnadas de afectividad (depresión) y serán propensos a avistar situaciones adversas futuras (ansiedad anticipatoria) o se abismaran en cavilaciones ansiogénas con evaluaciones pendulares sobre una misma cuestión (rumiaciones obsesivas). (Cía, 2006, p. 129)

Debido a ello, se esperaría que una vez regulada la biología, esta visión pudiera cambiar. En virtud de esto, podríamos postular que al momento de activarse el TOC surgiría una especie de "influencia desde el paradigma Biológico" que estaría dando una forma específica de funcionar en el mundo (Opazo, 2001). Ante esta hipótesis, parece interesante plantearse algún tipo de estudio comparativo entre pacientes que estén de alta en su tratamientos y los que no. De este modo, podríamos comprobar si existen algunas funciones que "permanecen" a pesar de esta activación biológica y separarlas de aquellos componentes más estructurales del sujeto.

Otro aspecto a considerar sería la edad de inicio del trastorno, aunque se estima que el inicio se produce entre los 20 y los 26 años, también se puede observar en niños entre 10 y 14 años (Cía, 2002, 2006). Si el trastorno inicia antes de que la personalidad esté completamente formada ¿Cómo afectará esto a la definición de sí mismo, a la identidad? ¿Cómo afectará a la forma en que se posiciona e interpreta el mundo? ¿Cómo afectará a las funciones del self?

Como consta en este artículo, en la presente investigación no se consideró esta variable. La investigación se realizó en personas mayores de 18 años, considerando que la personalidad ya estaría conformada al momento del inicio de los síntomas.

En relación a la función de identidad, es consistente que los pacientes con TOC y con alta conciencia de enfermedad, tiendan a ser personas neuróticas, capaces de responder de manera más o menos estable a la pregunta ¿Quién soy? Aún así, en la respuesta aparecen características negativas, lo que puede dar cuenta de bajo aprecio y bajo concepto de sí mismo. Así, si bien esta función se ve algo alterada, el sujeto logra realizar una apreciación de sí mismo con matices más negativos que positivos, y logrando mantener una forma estable de verse y percibirse a sí mismo. Esto unido a la percepción de la capacidad de realizar las cosas de forma adecuada en diferentes ámbitos, laboral, intelectual y sexual. Claro que en las áreas de pareja y social aparece mayor dificultad, quizás porque en dichas áreas entrarían en juego las variables afectivas, las que como se 
puede observar en la tabla $\mathrm{N}^{\circ} 5$ tienden a ser inestables y con predominio de afectos más negativos.

En relación a la función de significación, que correspondería a la "traducción de la experiencia dándole significado a ésta" (D'Orival, 2013, p. 23), se puede decir que en estudios anteriores surge como una de las funciones que más se encuentra alterada en sujetos con TOC (Downey, 2006). Consistente con esto, se puede observar en estos sujetos alta producción de ideas irracionales y distorsiones cognitivas, además de la tendencia a "ver" el mundo desde una perspectiva negativa. Aparece como elemento principal el "pensamiento rumiativo", que en este tipo de pacientes tienden a ser altamente desadaptativos y que les impiden poder funcionar en el mundo de manera adecuada, "presentan creencias muy sólidas sobre la probabilidad de que una determinada situación sea peligrosa para los demás o para sí mismos" (Hymann \& Pedrick, 2003, p. 160). Conjuntamente aparece el locus de control interno, llevándoles a "sentirse responsables" de todo lo que sucede a su alrededor, así "casi todos los pacientes temen que algo terrible pueda sucederles a ellos o a otros, por lo cual serán responsables" (Cía, 2002, p. 255).

Lo anterior también se acompaña de altos estándares de desempeño, combinados con la autoevaluación excesivamente crítica de sí mismos y la creciente preocupación por cometer errores. Esto puede llevarles a no movilizarse en el mundo, sintiendo que no son capaces de cumplir con lo que "deben" hacer, autoevaluándose de manera rígida y poco realista, no logrando cumplir con los altos estándares autoimpuestos. Para quienes hemos tratado a pacientes con TOC, no es extraño escuchar verbalizaciones en las que el sujeto afirma darse cuenta de lo poco racional de sus ideas; sin embargo, no lo pueden evitar. De esta manera, pareciera que la función de significación podría estar influenciada desde los aspectos biológicos, pudiendo producirse un estilo de "significación biológica", frente a lo cual, el sujeto no logra operar de manera racional ni clara. Es por esto que se puede decir que esta función aparece altamente alterada en este tipo de pacientes.

En cuanto a la función de autorganización, esta consiste en "la capacidad del sujeto de reconstruirse después del impacto que ocurre tras cada experiencia utilizando mecanismos internos de homeostasis" (D’Orival, 2013, p. 24). Según estudios previos, esta función se ve afectada de manera importante por el TOC (Downey, 2006). Como se puede observar aparece una alta perturbación frente a los estresores tanto internos como externos, junto con dificultad para volver a "re-armarse" internamente. Aparece predominancia de emociones por sobre sentimientos y alta inestabilidad emocional. Esto se encuentra acompañado de baja tolerancia frente las cosas que no resultan como ellos esperan, generando malestar interno y siéndoles muy difícil volver a un estado basal de neutralidad. El resultado es que tienden a quedarse "pegados" en las emociones displacenteras y no logran procesarlas de manera adecuada. Llama la atención que estos pacientes presenten locus de control interno para las supuestas amenazas, pero muestren locus de control externo al momento de controlar su propio malestar interno, surgiendo así los rituales como forma de mantener el orden donde no lo hay (dentro de sí mismo).

Adicionalmente, se puede observar que aparece alta frecuencia de eventos estresantes. Esta incidencia podría significar que las personas afectadas por el trastorno están más expuestas a diversos eventos estresantes o quizás, dadas las características del TOC, aquello que para cualquier persona que no lo sufra es solo un malestar, para personas con TOC, puede transformarse en algo extremadamente difícil. Así una experiencia simple como salir de casa, puede transformarse en una odisea en que aumenta la ansiedad e inseguridad, habiendo alta dificultad para poder re-establecer su equilibrio interior. Así, una paciente de 40 años, al salir de su casa y ya en la micro camino a la consulta psicológica, pasó por el lado de un cortejo fúnebre (que para ella era señal de contaminación de energías negativas), llegó con un nivel de desestructuración interna semejante al que ocurre frente a una catástrofe grave y de riesgo de vida, manteniendo este malestar por varias horas posteriores al hecho.

La función búsqueda de sentido se refiere a dar sentido y propósito a la existencia, respondiendo a la pregunta ¿Cuál es el propósito de mi vida? (D’Orival, 2013). En pacientes con TOC esta función puede verse interferida por las dificultades que aparecen tanto en el ámbito interpersonal como en la forma de ver e interpretar el mundo, ya que como se puede observar, estos pacientes son capaces de sentirse bien y capaces en el ámbito laboral, en donde parecieran poner su mayor esfuerzo y sentirse bien consigo mismo. No sería extraño que dichos pacientes centraran sus metas vitales en torno al trabajo y/o estudios, y aunque aparecen deseos de lograr tener pareja y de ser feliz, esto quedaría más en el imaginario Llama la atención que al momento de preguntarles por las expectativas en los procesos de terapia, se observa que la mayoría solicita "alivio sintomático", un porcentaje muy bajo desea comprender lo que les sucede. Esto podría dar cuenta de la forma más inmediatista de funcionar; podría deberse a que el 
TOC conlleva tan alto malestar que a nivel de sentido de vida, solo se desea no tener esta enfermedad, sin poder pensar más allá de ello. Como es conocido por quienes trabajan con este tipo de pacientes, estos se encuentran en una especie de círculo vicioso en donde desean hacer y ser algo diferente, y esto les genera ansiedad y temor. Ese temor lleva a que aumenten los síntomas del TOC, lo que les deja paralizados y sin poder salir de los síntomas. Es por ello que la función de búsqueda de sentido se vería bastante afectada por las características del TOC.

En relación a la función de conducción de vida, también se ve afectada. Si la función búsqueda de sentido es el "norte hacia donde se desea ir", la función conducción de vida sería "el chofer que llevaría a este lugar". Así la función consiste en movilizar conductas, desplegar esfuerzos permanentes, postergar impulsos, ejercer autocontrol y poder alcanzar las metas vitales y/o los propósitos de vida (FernándezÁlvarez \& Opazo, 2004). En los pacientes con TOC, esta función se ve altamente alterada, los síntomas del trastorno les impiden la movilización de las conductas en la dirección deseada, y aunque este tipo de personas pueden estar mucho tiempo dedicándose a los rituales y las ideas obsesivas, no presentan capacidad de dirigir dichos esfuerzos al cumplimiento de metas y/o propósitos. De esta forma, se puede observar que dedican tiempo y esfuerzo a "hacer las cosas perfectas", las cuales nunca están de la forma que desean, por lo que terminan abandonándolas o lo que es peor, surge como "distractor" la idea obsesiva. Esta desvía la atención de aquello que frustra y genera malestar, transformándolo en un malestar más "manejable" a través de rituales; todo ello producido por la idea. No es extraño encontrar personas con TOC que muestren altos niveles de frustración y que se vuelven irritables llegando a conductas agresivas como golpear puertas, tirar cosas, e incluso agredir a otros, como una forma de desprenderse de estos sentimientos de inadecuación e inseguridad.

Otro factor que pone la función de conducción de vida entre las alteradas por el TOC, sería el tiempo que cada persona ocupa en los rituales, dejando de lado las actividades de la vida cotidiana e incluso de la vida familiar. Por ejemplo, una paciente, para poder planchar la ropa, debía realizar marcas de cruces imaginarias con los dedos un número determinado de veces por cada prenda. Esto hacía que el planchado se extendiera por mucho más tiempo del que cualquier otra persona hubiese ocupado. Otro paciente, debía ducharse cada vez que entraba al baño, lo que le llevo a dejar de comer para evitar entrar al baño. Como consecuencia presentó anorexia grave que lo llevó al hospital y no pudo terminar su práctica profesional.

La función de toma de conciencia, que se refiere a los diferentes focos que intervienen en "el darse cuenta" en relación al entorno, la persona, la forma de funcionamiento, la historia personal, la capacidad de observación e introspección, la relación entre conducta y consecuencia y, en general, el autoconocimiento (D’Orival, 2013). En sujetos con TOC, esta función puede verse solo medianamente alterada, pues el sujeto logra darse cuenta de sí mismo, de lo que le sucede; sin embargo, no es capaz de utilizar esta información como una forma de producir el cambio. Aquí no es extraño que los pacientes verbalicen cosas como: "Sé que lo que pienso y hago está mal, que puede ser malo para mi hija pero igual debo hacerlo pues no lo puedo evitar" (Mujer, 40 años) o, en otro ejemplo, "gasto mucho detergente y he echado a perder 2 lavadoras por echarle tanto cloro, mi marido alega, pero no lo puedo evitar" (Mujer, 38 años).

Esta investigación es un intento de conceptualizar el TOC no solo desde la sintomatología, pues si se logra ir más allá en la comprensión de las dinámicas que subyacen a este trastorno, se podrían establecer guías de tratamiento centradas en la persona, no en la enfermedad. Así quizás, si los objetivos de tratamiento apuntaran a fortalecer aquellas funciones del self que se ven más alteradas, los pacientes podrían ver más allá de sus síntomas y contextualizarlos como formas desadaptativas de lidiar con aquello que les complica y molesta (formas más conocidas, con alto malestar, pero que de algún modo pueden controlar). Esto podría dar curso a aumentar la toma de conciencia y así, poder manejar los síntomas, como se hace en el trastorno de pánico. También se puede ayudar a fijar nortes orientadores que permitan tolerar el malestar del síntoma para lograr algo superior en la vida y de igual modo, se podría ayudar al sujeto a generar estrategias internas diferentes para poder lograr lo deseado.

\section{Referencias}

Antony, M., Downie, F. \& Swinson, R. (1998). Diagnostic issues and epidemiology in obsessive-compulsive disorder. En R. Swinson, M. Antony, S. Rachman \& M. Ritcher (Eds.), Obsessivecompulsive disorder: Theory, research and treatment (pp. 3-32). New York: Guilford Press.

American Psychological Association (2013). DSM V. Estados Unidos: Washington DC.

Araya, R., Rojas, G, Fritsch, R., Acuña, J. \& Lewis, G. (2001). Common mental disorders in Santiago, Chile. Prevalence and 
socio-demographic correlatos. The British Journal of Psychiatry, 178(3), 228-233. doi:10.1192/bjp.178.3.228

Asociación del trastorno obsesivo compulsivo de Andalucía (2015, 1 26). TOCAS. Recuperado de http://www.asociaciontocas.es/ eltoc.html

Bejer, T. (2010). Ficha de Evaluación Clínica Integral (FECI): características, validez y confiabilidad de las Escalas Clínicas. Actualizaciones en Psicoterapia Integrativa, 2, 38-52.

Cía, A. (2002). La ansiedad y sus trastornos. Buenos Aires: Polemos.

Cía, A. (2006). El trastorno Obsesivo compulsivo y su espectro. Buenos Aires: Polemos.

D’Orival, G. (2013). Alteración en la función de identidad en trastornos de personalidad a través de la descripción de uno mismo: un estudio preliminar (Tesis de maestría inédita). Santiago, Chile.

Downey, P. (2006). Descripción del Trastorno Obsesivo Compulsivo: Una Mirada desde el Modelo Integrativo Supraparadigmático (Tesis de maestría inédita). Universidad Adolfo Ibáñez e Instituto Chileno de Psicoterapia Integrativa, Santiago, Chile.

Fernández-Álvarez, H. \& Opazo, R. (2004). La Integración en psicoterapia: Manual Práctico. Barcelona: Paidós.
Hymann, B. \& Pedrick, C. (2003). Guía Práctica del trastorno obsesivo compulsivo: Pistas para su liberación. España: Desclée De Brouwer.

Marks, I., Hodgson, R. \& Rachman, S. (1975). Treatment of chronic obsessive-compulsive neurosis by in vivo exposure: A tow-year follow-up and issue in treatment. The British Journal of Psychiatric, 127(4), 349-364. doi:10.1192/bjp.127.4.349

Nicolini, H., Weissbecker, K., Mejia, J. \& de Carmona, M. (1993). Family study of obsessive compulsive disorder in a Mexican Population. Archives of Medical Research, 24(2), 193-198.

Opazo, R. (2001). Psicoterapia Integrativa. Delimitación Clínica. Santiago, Chile: ICPSI.

Ruiz, A. (1994). Qué nos pasa cuando estamos deprimido. Cómo entender y cómo cambiar nuestras experiencias depresivas a través de la terapia cognitiva. Santiago, Chile: Instituto de Terapia Cognitiva.

Torres, A., Ferrao, Y., Chavit, R., Diniz, J., Costa, D., do Rosário, M., ... \& Fontenelle, L. (2014). Panic Disorder and agoraphobia in OCD patients: clinical profile and possible treatment implications. Comprehensive Psychiatryc, 55(3), 588-597. doi: 10.1016/j. comppsych.2013.11.017 